\title{
RESULTS OF TESTING GYPSUM PRODUCTS*
}

By W. E. EmLey and C. F. Faxon

In an effort to write standard specifications for gypsum, many difficulties have been encountered. Probably the greatest source of confusion was the attempt to adopt verbatim the standard methods of test which were in use in the examination of other similar materials. Experience showed the futility of this procedure, and finally compelled the invention of new methods of test, designed especially for gypsum. After considerable deliberation and experiment, a number of methods of test were finally agreed upon and adopted as tentative. ${ }^{1}$

It then developed that no one had had enough experience with these new methods to be able to predict what numerical results they would give. For example, take the tensile strength. This property of gypsum was pretty well known when measured by any one of several different methods. The method adopted, however, introduced certain innovations, so that no one could foretell just what tensile strength a given sample of material would have when tested by this new method.

Obviously some information about these numerical results was essential, in order that specifications could be intelligently written. It would be absurd to specify a tensile strength of 300 pounds per square inch, and then discover that either all or none of the gypsum on the market met the requirement.

Accordingly the Bureau of Standards undertook to test a number of commercial samples. This article is a compilation of the results obtained. The tests were carried out on 43 samples, made by three different manufacturers. Of these, 25 were shipped to us direct from the factory, packed in air-tight glass containers; 8 came direct from the factory, in the usual commercial package; and ro were obtained from dealers.

* Received Sept. I, 1920.

${ }^{1}$ American Society for Testing Materials, C-26-19T. 
While an attempt was made to follow the methods of test cited above, certain changes and additions were found advisable and were accordingly made. A brief description of the methods is as follows:

I. Chemical Analysis.-Lime,- sulphuric anhydride, carbon dioxide, and loss on ignition, were determined by the usual methods of chemical analysis. ${ }^{1}$ These constituents were then combined as follows: The amount of lime required to combine with the carbon dioxide was found by multiplying the per cent carbon dioxide by $56 / 44$. The per cent lime present as carbonate was deducted from the total per cent lime. The per cent water was found by subtracting the per cent carbon dioxide from the per cent loss on ignition. From the figures for lime, sulphuric anhydride, and water, the maximum possible content of calcined gypsum $\left(\mathrm{CaSO}_{4} \cdot{ }^{1} /{ }_{2} \mathrm{H}_{2} \mathrm{O}\right)$ was calculated, using the ratios $56: 80: 9$. Usually two, and always one of these three ingredients was found to be in excess of these ratios. This was taken to indicate the presence of some foreign material, such as calcium hydroxide, magnesium sulphate, anhydrous calcium sulphate, etc.

2. Normal Consistency.-This is the number of cubic centimeters of water which must be added to Ioo grams of dry material to produce a paste of standard "wetness." It was determined by means of the Southard viscosimeter, ${ }^{2}$ the standard wetness being such that the final radius of the pat was $9.6 \mathrm{cms}$.

3. Time of Set.--This is measured by means of a Vicat needle, on material of normal consistency. It is the elapsed time from when the sample is added to the water to when the needle fails to penetrate to the bottom of the pat.

4. Fineness. -This is expressed as the per cent by weight of the material separated by six sieves of different meshes. The sieves used were the Nos. 8, 14, 28, 48, 100 and 200. In general, the material could be screened dry through Nos. 8 and $\mathrm{I}_{4}$, but had to be washed with kerosene in order to get clean separations on the finer sieves.

${ }^{1}$ Hillebrand, "Analysis of Silicate and Carbonate Rocks," U. S. Geol. Sur., Bull. 700.

${ }^{2}$ A. S. T. M., C26-I9T. 
5. Compressive Strength.--Three cylinders, 2 inches in diameter by 4 inches high, were made of paste of normal consistency. They were removed from the molds as soon as they were hard enough to handle ( $\mathrm{r}$ to 24 hours), stored in the air in the room for one week, and tested. The. results are expressed as pounds square inch.

\section{TABLE I}

$\begin{aligned} \text { Lab. No. } & \text { Trade name of material } \\ \text { I } & \text { Unretarded gauging plaster } \\ 2 & \text { Molding plaster } \\ \text { 10 } & \text { Unretarded gauging plaster } \\ \text { I I } & \text { Molding plaster } \\ \text { 19 } & \text { Unretarded gauging plaster } \\ 20 & \text { Molding plaster } \\ 26 & \text { Stucco } \\ 28 & \text { Molding plaster } \\ 29 & \text { Reground stucco } \\ 31 & \text { Stucco } \\ 32 & \text { Stucco } \\ 34 & \text { Plaster of Paris F } \\ 35 & \text { Plaster of Paris FFF } \\ 37 & \text { Windsor cement FFF } \\ 43 & \text { Potters plaster } \\ \text { Ave. } & \end{aligned}$

Class

Calcined gypsum only
Retarding gauging plaster
Cement plaster, unsanded, not fibered
I 2 Retarded gauging plaster
I3 Cement plaster, unsanded, not fibered
21 Retarded gauging plaster
22 Cement plaster, unsanded, not fibered
38 Ready finish
4I Superfine Windsor cement

Ave.

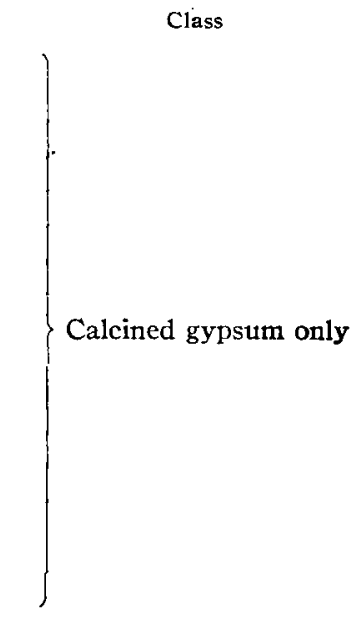

5 Cement plaster, unsanded, fibered

16 Retarded fibered cement plaster

23 Fibered cement plaster

27 Fibered plaster

33 Fibered plaster

36 Windsor cement, neat

Ave.

Calcined gypsum plus re tarder

Calcined gypsum plus retarder plus fiber 


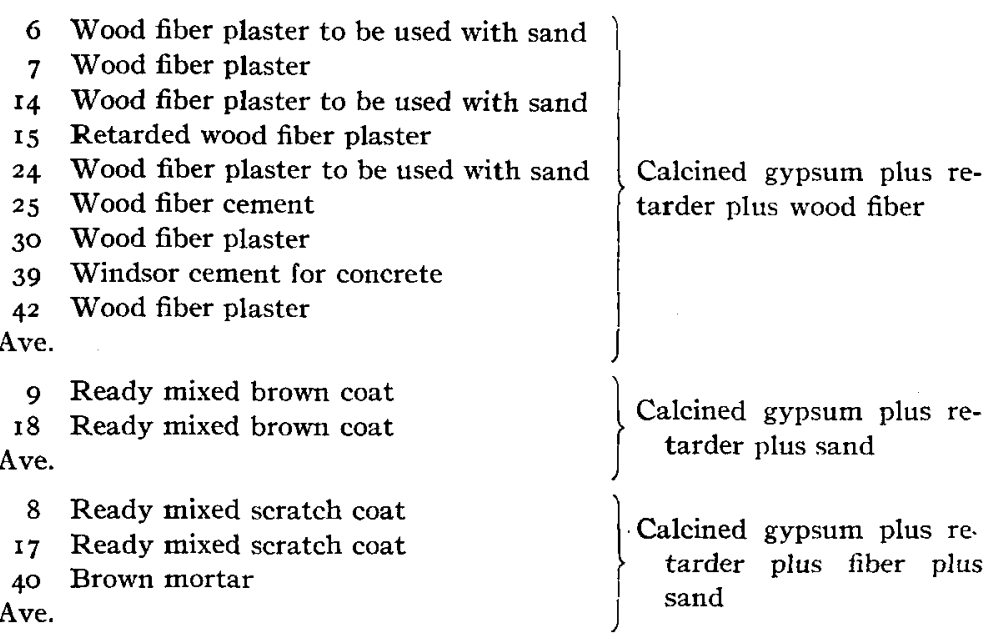

TABLE 2

\begin{tabular}{|c|c|c|c|c|c|c|}
\hline \multirow[b]{3}{*}{ Lab No. } & \multicolumn{6}{|c|}{ Chemical analysis } \\
\hline & \multicolumn{4}{|c|}{ Found } & \multicolumn{2}{|c|}{ Calculated } \\
\hline & $\mathrm{CaO}$ & $\mathrm{CO}_{2}$ & $\mathrm{SO}_{3}$ & $\begin{array}{c}\text { Loss on } \\
\text { ignition }\end{array}$ & $\begin{array}{l}\text { Calcined } \\
\text { gypsumi }\end{array}$ & $\begin{array}{c}\text { Constituents } \\
\text { in excess }\end{array}$ \\
\hline I $\ldots \ldots \ldots$ & 37.60 & $4.2 \mathrm{I}$ & 48.73 & 10.40 & 83.50 & $\mathrm{SO}_{3} . \mathrm{H}_{2} \mathrm{O}$ \\
\hline $2 \ldots \ldots \ldots$ & 37.20 & 4.22 & 49.00 & 10.70 & 82.40 & $\mathrm{SO}_{3} \cdot \mathrm{H}_{2} \mathrm{O}$ \\
\hline 10. $\ldots \ldots \ldots$ & 34.80 & 12.53 & 36.80 & 16.20 & 48.77 & $\mathrm{SO}_{3} \cdot \mathrm{H}_{2} \mathrm{O}$ \\
\hline II $\ldots \ldots \ldots$ & 35.28 & I I . OO & 38.55 & 16.40 & $55 \cdot 10$ & $\mathrm{SO}_{3} \cdot \mathrm{H}_{2} \mathrm{O}$ \\
\hline $19 \ldots \ldots \ldots$ & 38.12 & 0.56 & 53.50 & 8.00 & 97.02 & $\mathrm{CaO} \mathrm{H}_{2} \mathrm{O}$ \\
\hline $20 \ldots \ldots \ldots$ & 38.24 & .74 & 53.64 & 8.00 & 86.60 & $\mathrm{SO}_{3} . \mathrm{H}_{2} \mathrm{O}$ \\
\hline $26 \ldots \ldots \ldots$ & 38.20 & . IO & 54.00 & 7.60 & 97.88 & $\mathrm{CaO} \cdot \mathrm{H}_{2} \mathrm{O}$ \\
\hline $28 \ldots \ldots \ldots$ & 37.94 & .10 & 53.90 & $7 \cdot 70$ & 97.69 & $\mathrm{CaO} \cdot \mathrm{H}_{2} \mathrm{O}$ \\
\hline $29 \ldots \ldots \ldots$ & 35.80 & 2.40 & 47.80 & 9.20 & 84.83 & $\mathrm{SO}_{3} \cdot \mathrm{H}_{2} \mathrm{O}$ \\
\hline $31 \ldots \ldots \ldots$ & 37.44 & 0.60 & 51.90 & 7.90 & 94.07 & $\mathrm{CaO} . \mathrm{H}_{2} \mathrm{O}$ \\
\hline $32 \ldots \ldots \ldots$ & 37.10 & 4.10 & 48.70 & 10.60 & 82.54 & $\mathrm{SO}_{3} . \mathrm{H}_{2} \mathrm{O}$ \\
\hline $34 \ldots \ldots \ldots$ & 38.00 & I.OO & 51.65 & $7 \cdot 53$ & 93.65 & $\mathrm{CaO} . \mathrm{H}_{2} \mathrm{O}$ \\
\hline $35 \ldots \ldots \ldots$ & 38.26 & 0.62 & 53.70 & 6.91 & $97 \cdot 10$ & $\mathrm{SO}_{3} . \mathrm{H}_{2} \mathrm{O}$ \\
\hline $37 \ldots \ldots \ldots$ & 38.13 & .53 & 53.80 & 6.94 & 97.00 & $\mathrm{SO}_{3} . \mathrm{H}_{2} \mathrm{O}$ \\
\hline $43 \ldots \ldots \ldots$ & $3^{8.90}$ & .70 & 53.60 & 7.55 & $97 \cdot 15$ & $\mathrm{CaO} . \mathrm{H}_{2} \mathrm{O}$ \\
\hline Ave........ & $37 \cdot 41$ & 2.89 & 49.98 & 9.44 & 87.02 & \\
\hline $3 \ldots \ldots \ldots$ & 37.04 & $4 \cdot 53$ & 48.28 & 10.90 & $83 \cdot 50$ & $\mathrm{SO}_{3} \cdot \mathrm{H}_{2} \mathrm{O}$ \\
\hline $4 \ldots \ldots \ldots$ & 36.72 & $4 \cdot 37$ & 47.90 & I0. 75 & 80.60 & $\mathrm{SO}_{3} \cdot \mathrm{H}_{2} \mathrm{O}$ \\
\hline I2 $\ldots \ldots \ldots$ & 34.52 & 10.02 & 36.49 & 17.35 & 56.35 & $\mathrm{SO}_{3} . \mathrm{H}_{2} \mathrm{O}$ \\
\hline$I_{3} \ldots \ldots \ldots$ & 34.84 & I2.33 & $37 \cdot 56$ & I 6.55 & $49 \cdot 5^{8}$ & $\mathrm{SO}_{3} \cdot \mathrm{H}_{2} \mathrm{O}$ \\
\hline $2 r \ldots \ldots \ldots$ & 38.60 & $0.8 \mathrm{I}$ & 53.30 & 7.55 & 96.60 & $\mathrm{CaO} \cdot \mathrm{H}_{2} \mathrm{O}$ \\
\hline $22 \ldots \ldots \ldots$ & 38.28 & .84 & 53.74 & 8.10 & 96.40 & $\mathrm{SO}_{3} \cdot \mathrm{H}_{2} \mathrm{O}$ \\
\hline
\end{tabular}


TABLE 2 (Continued)

\begin{tabular}{|c|c|c|c|c|c|c|}
\hline \multirow[b]{3}{*}{ Lab. No. } & \multicolumn{6}{|c|}{ Chemical analysis } \\
\hline & \multicolumn{4}{|c|}{ Found } & \multicolumn{2}{|c|}{ Calculated } \\
\hline & $\mathrm{CaO}$ & $\mathrm{CO}_{2}$ & $\mathrm{SO}_{3}$ & $\begin{array}{l}\text { Loss on } \\
\text { ignition }\end{array}$ & $\begin{array}{c}\text { Calcined } \\
\text { gypsumm }\end{array}$ & $\begin{array}{c}\text { Constituents } \\
\text { in excess }\end{array}$ \\
\hline $38 \ldots \ldots \ldots$ & $39 \cdot 20$ & 2.83 & I $5 \cdot 55$ & $14 \cdot 20$ & 28.20 & $\mathrm{CaO} . \mathrm{H}_{2} \mathrm{O}$ \\
\hline $41 \ldots \ldots \ldots$ & $35 \cdot 72$ & 15.20 & 35.90 & 20.05 & $4^{2} \cdot 3^{8}$ & $\mathrm{SO}_{3} \cdot \mathrm{H}_{2} \mathrm{O}$ \\
\hline Ave....... & 36.86 & 6.37 & $4 \mathrm{I} \cdot 09$ & I 3.18 & 66.70 & \\
\hline $5 \ldots \ldots \ldots$ & 36.96 & 4.73 & 47.83 & I I . I 5 & 80.20 & $\mathrm{SO}_{3} \cdot \mathrm{H}_{2} \mathrm{O}$ \\
\hline $16 \ldots \ldots \ldots$ & 35.00 & I 2.16 & 37.90 & I $7 \cdot 15$ & $50 \cdot 56$ & $\mathrm{SO}_{3} \cdot \mathrm{H}_{2} \mathrm{O}$ \\
\hline $23 \ldots \ldots \ldots$ & 36.88 & 0.68 & $52 \cdot 30$ & $7 \cdot 35$ & $93 \cdot 30$ & $\mathrm{SO}_{3} \cdot \mathrm{H}_{2} \mathrm{O}$ \\
\hline $27 \ldots \ldots \ldots$ & 38.24 & .40 & 53.90 & $7 \cdot 90$ & 97.68 & $\mathrm{SO}_{3} \cdot \mathrm{H}_{2} \mathrm{O}$ \\
\hline $33 \ldots \ldots \ldots$ & $37 \cdot 70$ & .10 & $53 \cdot 40$ & 7.80 & 96.79 & $\mathrm{CaO} \cdot \mathrm{H}_{2} \mathrm{O}$ \\
\hline $36 \ldots \ldots \ldots$ & 36.88 & I. 40 & 48.50 & 8.18 & 87.95 & $\mathrm{CaO} \cdot \mathrm{H}_{2} \mathrm{O}$ \\
\hline Ave........ & 36.94 & 3.24 & $4^{8.97}$ & 9.92 & $84 \cdot 4 \mathrm{I}$ & $\mathrm{CaO} \cdot \mathrm{H}_{2} \mathrm{O}$ \\
\hline $6 \ldots \ldots \ldots$ & 36.92 & $4 \cdot 36$ & 48.52 & 10.70 & 81,20 & $\mathrm{SO}_{3} \cdot \mathrm{H}_{2} \mathrm{O}$ \\
\hline $7 \ldots \ldots \ldots$ & 37.00 & $4 \cdot 41$ & 48.59 & IO. 50 & $8 \mathrm{I} .25$ & $\mathrm{SO}_{3} \cdot \mathrm{H}_{2} \mathrm{O}$ \\
\hline I $4 \ldots \ldots \ldots$ & $34 \cdot 96$ & 12.64 & $37 \cdot 32$ & $17 \cdot 35$ & 48.86 & $\mathrm{SO}_{3} \cdot \mathrm{H}_{2} \mathrm{O}$ \\
\hline I $5 \ldots \ldots \ldots$ & $35 \cdot 12$ & I 1.90 & 38.10 & 17.40 & $5 \mathrm{I} .7 \mathrm{O}$ & $\mathrm{SO}_{3} \cdot \mathrm{H}_{2} \mathrm{O}$ \\
\hline $24 \ldots \ldots \ldots$ & 38.20 & 0.74 & $53 \cdot 64$ & 7.90 & $96 \cdot 47$ & $\mathrm{SO}_{3} \cdot \mathrm{H}_{2} \mathrm{O}$ \\
\hline $25 \ldots \ldots \ldots$ & 38.08 & .82 & 53.06 & 7.95 & $95 \cdot 95$ & $\mathrm{SO}_{3} \cdot \mathrm{H}_{2} \mathrm{O}$ \\
\hline $30 \ldots \ldots \ldots$ & 36.14 & 2.50 & 48.40 & $9 \cdot 40$ & $85 \cdot 34$ & $\mathrm{SO}_{3} \cdot \mathrm{H}_{2} \mathrm{O}$ \\
\hline $39 \ldots \ldots \ldots$ & $27 \cdot 4 \mathrm{I}$ & 1. 64 & $27 \cdot 70$ & 9.25 & 50.22 & $\mathrm{CaO} \cdot \mathrm{H}_{2} \mathrm{O}$ \\
\hline $42 \ldots \ldots \ldots$ & $35 \cdot 40$ & $\mathbf{I} \cdot 3 \mathbf{I}$ & 46.40 & 8.60 & $84 \cdot 10$ & $\mathrm{CaO} . \mathrm{H}_{2} \mathrm{O}$ \\
\hline Ave........ & $35 \cdot 47$ & $4 \cdot 48$ & 44.64 & II.OI & 75.01 & $\mathrm{CaO} \cdot \mathrm{H}_{2} \mathrm{O}$ \\
\hline $9 \ldots \ldots \ldots$ & 18.20 & 10.86 & I 3.49 & 13.10 & II .27 & $\mathrm{SO}_{3} \cdot \mathrm{H}_{2} \mathrm{O}$ \\
\hline I $8 \ldots \ldots \ldots$ & 25.60 & $15 \cdot 70$ & $\times 3.68$ & 17.80 & 14.50 & $\mathrm{SO}_{3} \cdot \mathrm{H}_{2} \mathrm{O}$ \\
\hline Ave........ & $2 I \cdot 90$ & $\mathrm{I} 3.28$ & $13 \cdot 58$ & $15 \cdot 45$ & I 2.88 & \\
\hline $8 \ldots \ldots \ldots$ & 18.52 & 10.32 & I 4.80 & 12.75 & 13.92 & $\mathrm{SO}_{3} \cdot \mathrm{H}_{2} \mathrm{O}$ \\
\hline $17 \ldots \ldots \ldots$ & 24.80 & 13.86 & I 5.84 & 16.70 & 18.49 & $\mathrm{SO}_{3} \cdot \mathrm{H}_{2} \mathrm{O}$ \\
\hline $40 \ldots \ldots \ldots$ & 8.76 & 0.47 & 10.80 & 3.09 & 19.58 & $\mathrm{CaO} \cdot \mathrm{H}_{2} \mathrm{O}$ \\
\hline Ave....... & I $7 \cdot 36$ & 8.22 & $13.8 \mathrm{I}$ & 10.85 & $17 \cdot 33$ & \\
\hline
\end{tabular}

TABLE 3

\begin{tabular}{|c|c|c|c|c|c|c|c|}
\hline \multirow[b]{2}{*}{ Lab. No. } & \multicolumn{6}{|c|}{ Fineness } & \multirow[b]{2}{*}{$\underset{200}{\text { Through }}$} \\
\hline & On 8 & $8-14$ & 14.28 & $28-48$ & $48-100$ & $100-200$ & \\
\hline$x \ldots \ldots \ldots$ & $\ldots$ & O. I & $0 . I$ & 0.9 & 3.9 & 15.4 & 79.6 \\
\hline $2 \ldots \ldots$ & $\ldots$ & $\ldots$ & .2 & I. 1 & 4. I & 8.2 & 86.4 \\
\hline $10 \ldots \ldots \ldots$ & $\ldots$ &. $\mathrm{I}$ & .3 & 4.6 & 7.0 & 11.5 & 76.5 \\
\hline $11 \ldots$ & $\ldots$ &.$I$ &.$I$ & .3 & 0.7 & 6.8 & 92.0 \\
\hline $19 \ldots \ldots$ & $\cdots$ &.$I$ & .3 & 3.0 & 24.0 & 26.7 & $45 \cdot 9$ \\
\hline $20 \ldots \ldots$ & $\ldots$ & $\ldots$ &. $\mathrm{I}$ & 0.5 & 6.3 & 23.2 & 69.9 \\
\hline
\end{tabular}




\begin{tabular}{|c|c|c|c|c|c|c|c|}
\hline $26 \ldots \ldots \ldots$ & $\ldots$ & $\cdots$ & 3 & 4.0 & 15.6 & 29.7 & 50.4 \\
\hline $28 \ldots \ldots \ldots$ & $\ldots$ & $\cdots$ &. $\mathbf{I}$ & 0.3 & 4.8 & I 5.6 & 79.2 \\
\hline $29 \ldots \ldots \ldots$ & $\cdots$ & $\cdots$ & .7 & 3.8 & 8.5 & $20 \cdot 3$ & 66.7 \\
\hline $31 \ldots \ldots \ldots$ & $\ldots$ & $\cdots$ &. $\mathbf{I}$ & o. 4 & I. I & $9 . I$ & 89.3 \\
\hline $32 \ldots \ldots \ldots$ & $\ldots$ & $\ldots$ & .2 & I $\cdot 3$ & $3 \cdot 1$ & 8.2 & 87.2 \\
\hline $34 \ldots \ldots \ldots$ & 0.3 & 0.7 & 2.5 & 8.8 & 16.4 & I I . I & 60.2 \\
\hline $35 \ldots \ldots \ldots$ & $\cdots$ & $\cdots$ & $\cdots$ & 0.2 & $9 \cdot 3$ & I 3.4 & 77.1 \\
\hline $37 \ldots \ldots \ldots$ & $\ldots$ & $\ldots$ & $\cdots$ &. $\mathrm{I}$ & 6.1 & $9 \cdot 7$ & 84.1 \\
\hline $43 \ldots \ldots \ldots$ & $\cdots$ & $\cdots$ & $\cdots$ & 3 & $4 \cdot 9$ & 13.2 & 81.6 \\
\hline Ave........ & 0.0 & o. $\mathbf{I}$ & 0.3 & 2.0 & $7 \cdot 7$ & I $4 \cdot 5$ & $75 \cdot 1$ \\
\hline $3 \ldots \ldots \ldots$ & $\cdots$ & $\cdots$ & 0.2 & 0.9 & 3.1 & I0. 7 & $85 \cdot 1$ \\
\hline $4 \ldots \ldots \ldots$ & $\ldots$ & $\cdots$ &. $\mathrm{I}$ & I. 3 & $4 \cdot I$ & 12.4 & 82.1 \\
\hline I $2 \ldots \ldots \ldots$ & $\cdots$ & $0 . \mathbf{I}$ & $\cdot 3$ & $4 \cdot I$ & 8.9 & .8 .8 & 77.8 \\
\hline $13 \ldots \ldots \ldots$ & $\cdots$ & $\cdots$ & $\mathbf{I}$ & 0.2 & I. 2 & $4 \cdot 7$ & 93.8 \\
\hline $2 \mathbf{I} \ldots \ldots \ldots$ & $\ldots$ & $\ldots$ & .2 & $2 \cdot 5$ & 18.0 & 28.7 & 50.6 \\
\hline $22 \ldots \ldots \ldots$ & $\cdots$ & $\ldots$ & $\cdot 3$ & 2.2 & I 8.6 & $2 \mathrm{I} \cdot 5$ & $57 \cdot 4$ \\
\hline $3^{8} \ldots \ldots \ldots$ & $\ldots$ & $\ldots$ & $\cdots$ & 0.2 & 2.6 & $7 \cdot 7$ & $89 \cdot 5$ \\
\hline $41 \ldots \ldots \ldots$ & $\ldots$ & $\cdots$ & $\ldots$ & I. 7 & $\mathrm{I} 4 \cdot \mathrm{I}$ & 10. 3 & $73 \cdot 9$ \\
\hline Ave........ & 0.0 & 0.0 & O. I & I. 6 & 8.8 & 13.1 & 76.3 \\
\hline $5 \ldots \ldots \ldots$ & 0.1 & o. I & o. $\mathbf{I}$ & 0.9 & $3 \cdot 3$ & 10.8 & $84 \cdot 7$ \\
\hline $16 \ldots \ldots \ldots$ & I I &. $\mathbf{I}$ & $\mathrm{I}$ & .2 & 0.8 & 6.4 & $92 \cdot 3$ \\
\hline $23 \ldots \ldots \ldots$ & I I &. $\mathbf{I}$ & .2 & 1.7 & $I_{4} .8$ & 21.9 & $6+2$ \\
\hline $27 \ldots \ldots \ldots$ & $\ldots$ & $\ldots$ & .2 & 0.9 & 2.8 & 17.0 & 79.1 \\
\hline $33 \ldots \ldots \ldots$ & $\ldots$ & $\ldots$ & .3 & I. 5 & $4 \cdot 8$ & 28.7 & $64 \cdot 7$ \\
\hline $36 \ldots \ldots \ldots$ & $0 . I$ & 0.4 & $2 \cdot 3$ & $13 \cdot 5$ & 16.8 & 9.6 & $57 \cdot 4$ \\
\hline Ave...... & . I & I & 0.5 & $3 \cdot r$ & $7 \cdot 2$ & I $5 \cdot 9$ & $73 \cdot 2$ \\
\hline $6 \ldots \ldots$. & 0.5 & 0.4 & 0.5 & I. 7 & 3.8 & $9 \cdot 7$ & $83 \cdot 4$ \\
\hline $7 \ldots \ldots \ldots$ & .7 & .3 & $\cdot 3$ & I. 7 & $4 \cdot 0$ & 10.7 & $82 \cdot 3$ \\
\hline $14 \ldots \ldots \ldots$ & $\cdot 4$ & .2 & .4 & $4 \cdot 0$ & 8.9 & I I . O & $75 \cdot \mathrm{I}$ \\
\hline $15 \ldots \ldots \ldots$ & .4 & .2 & .4 & $3 \cdot 7$ & 8.6 & $9 \cdot 5$ & 77.2 \\
\hline $24 \ldots \ldots \ldots$ & .8 & .5 & .6 & $2 \cdot 7$ & $17 \cdot 5$ & 19.3 & 58.6 \\
\hline $25 \ldots \ldots \ldots$ & .4 &.$I$ & .4 & .2 .6 & 19.5 & 22.6 & $54 \cdot 4$ \\
\hline $30 \ldots \ldots \ldots$ &. $\mathbf{I}$ & .2 & 1.2 & $3 \cdot 5$ & $9 \cdot 7$ & $22 \cdot 4$ & 62.9 \\
\hline $39 \ldots \ldots \ldots$ & I. 5 & $4 \cdot 2$ & $7 \cdot 5$ & 16.0 & $17 \cdot 5$ & 6.6 & 46.7 \\
\hline $42 \ldots \ldots \ldots$ & 0.3 & 0.6 & I , I & 8.5 & $23 \cdot 5$ & 9.4 & 56.6 \\
\hline Ave....... & .6 & .8 & I $\cdot 4$ & $4 \cdot 9$ & 12.6 & I $3 \cdot 5$ & 66.4 \\
\hline$\ldots \ldots$ & O. I & 8.9 & 10. 9 & I 8.0 & $25 \cdot 4$ & 16.7 & 20.0 \\
\hline $18 \ldots \ldots \ldots$ & .8 & 21.2 & 21.6 & Io. 8 & II. 4 & 3.8 & 30.4 \\
\hline Ave........ & .4 & 15.0 & 16.2 & 14.4 & 18.4 & IO. 2 & 25.2 \\
\hline $8 \ldots \ldots \ldots$ & 0.2 & 10.3 & I I . 3 & 12.8 & $2 I .2$ & $\mathbf{1} 3 \cdot 7$ & 30.5 \\
\hline I $7 \ldots \ldots$ & 3.6 & 30.0 & I 2.8 & 6.5 & IO. 0 & $4 \cdot 4$ & $32 \cdot 7$ \\
\hline $40 \ldots \ldots \ldots$ & 0.5 & $5 \cdot 3$ & 10.2 & 31.4 & 26.0 & 8.0 & 18.6 \\
\hline Ave........ & i. 4 & $15 \cdot 2$ & I I. 4 & .16 .9 & I9. I & 8.7 & $27 \cdot 3$ \\
\hline
\end{tabular}




\begin{tabular}{|c|c|c|c|c|c|c|}
\hline & & $\begin{array}{c}\text { TABLE } 4 \\
\text { Yield }\end{array}$ & & & & \\
\hline $\begin{array}{c}\text { Compressive } \\
\text { strength }\end{array}$ & $\begin{array}{l}\text { Lbs. } \\
\text { paste } \\
\text { per } \\
\text { cu. ft. }\end{array}$ & $\begin{array}{l}\text { Lbs. dry } \\
\text { mater- } \\
\text { ial per } \\
\text { cu. ft. } \\
\text { paste }\end{array}$ & $\begin{array}{c}\text { Lbs. set } \\
\text { material } \\
\text { per } \\
\text { cu. ft. s }\end{array}$ & $\begin{array}{l}\text { T'ensile } \\
\text { strength }\end{array}$ & $\begin{array}{l}\text { Normal } \\
\text { con- } \\
\text { sistency }\end{array}$ & $\begin{array}{l}\text { Time } \\
\text { of set }\end{array}$ \\
\hline $1 \ldots \ldots \ldots 1460$ & 106 & 64 & 76 & 254 & 66.1 & $2 I$ \\
\hline $2 \ldots \ldots \ldots$ 1315 & 107 & 64 & 75 & 775 & 66.9 & $2 \mathrm{I}$ \\
\hline I0. . . . . . I700 & I 13 & 77 & 88 & 333 & 46.6 & I 2 \\
\hline II $\ldots \ldots \ldots$ I 570 & 107 & 66 & 79 & 270 & 56.2 & 7 \\
\hline $19 \ldots \ldots \ldots \ldots$ II & IOI & 58 & 68 & 244 & $73 \cdot 2$ & I I \\
\hline $20 \ldots \ldots \ldots \ldots 1060$ & 102 & 58 & 68 & 220 & $75 \cdot 0$ & I9 \\
\hline $26 \ldots \ldots \ldots 1550$ & I I 3 & 74 & 82 & 413 & 53.0 & I4 \\
\hline $28 \ldots \ldots \ldots \ldots$ I 580 & I I 2 & 72 & 81 & 387 & 56.0 & 22 \\
\hline $29 \ldots \ldots \ldots 1956$ & II 4 & 77 & 89 & 357 & 48.0 & 16 \\
\hline $31 \ldots \ldots \ldots 1935$ & 107 & 68 & 78 & 394 & 58.0 & 8 \\
\hline $32 \ldots \ldots \ldots \ldots 1875$ & 110 & 72 & 83 & 444 & 51.8 & 12 \\
\hline $34 \ldots \ldots \ldots 2200$ & 114 & 76 & 85 & 437 & 50.0 & 13 \\
\hline $35 \ldots \ldots \ldots$ I630 & 111 & 69 & 79 & 280 & 60.2 & 9 \\
\hline $37 \ldots \ldots \ldots 2285$ & 116 & 75 & 83 & 299 & $5 I \cdot 5$ & $8: 00$ \\
\hline $43 \ldots \ldots \ldots r 720$ & 92 & 57 & 78 & 270 & 61.0 & 9:0o \\
\hline Ave........ 1665 & 108 & 62 & 80 & 325 & 58.2 & 1 3:00 \\
\hline $3 \ldots \ldots \ldots \quad 1295$ & 107 & 65 & 77 & 273 & $64 \cdot 3$ & $53: 00$ \\
\hline $4 \ldots \ldots \ldots .925$ & 105 & 63 & 74 & 236 & 66.0 & I I : 56 \\
\hline $12 \ldots \ldots \ldots \ldots I_{42}$ & I I I & 76 & 87 & 290 & 46.2 & $2: 54$ \\
\hline r3 $\ldots \ldots \ldots 6630$ & I 12 & 74 & 85 & 2 I 8 & 51.9 & I $7: 23$ \\
\hline $21 \ldots \ldots \ldots 1280$ & 103 & 62 & 73 & 272 & $65 \cdot 4$ & I. 58 \\
\hline $22 \ldots \ldots \ldots \ldots$ I0OO & 103 & 62 & 73 & $26 \mathrm{I}$ & $65 \cdot c$ & I3:07 \\
\hline $38 \ldots \ldots \ldots \ldots$ & 93 & 50 & 54 & 37 & $85 \cdot 5$ & $1: 00$ \\
\hline $4 r \ldots \ldots \ldots 1400$ & 120 & 83 & 87 & I 29 & 44.6 & $5: 37$ \\
\hline Ave......... roio & 107 & 67 & 76 & 215 & $6 \mathrm{I} \cdot \mathrm{I}$ & $6: 4 I$ \\
\hline $5 \ldots \ldots \ldots \quad 555$ & 104 & 63 & 73 & 182 & $65 \cdot 7$ & I $4: 15$ \\
\hline $16 \ldots \ldots \ldots$ IIOO & I ro & 73 & 80 & 254 & $5 \mathrm{I} \cdot 2$ & $25: 24$ \\
\hline $23 \ldots \ldots \ldots \ldots$ I030 & 106 & 66 & 75 & 269 & 61.0 & $13: 09$ \\
\hline $27 \ldots \ldots \ldots$ I 730 & 107 & 69 & 81 & 379 & $55 \cdot 5$ & $6: 34$ \\
\hline $33 \ldots \ldots \ldots$ I 475 & $1 \times 5$ & 75 & 83 & 400 & $53 \cdot 3$ & $8: 50$ \\
\hline $36 \ldots \ldots \ldots 800$ & 107 & 70 & 80 & I 86 & $5 I .6$ & $19: 42$ \\
\hline Ave......... I I I 5 & 108 & 69 & 79 & 278 & 56.4 & I $4: 39$ \\
\hline $6 \ldots \ldots \ldots 835$ & 103 & 62 & 73 & 222 & $65 \cdot 4$ & I $8: 35$ \\
\hline $7 \ldots \ldots \ldots 670$ & I03 & 62 & 74 & 195 & 66.3 & $6: 17$ \\
\hline $\mathbf{1} 4 \ldots \ldots \ldots \quad 78_{5}$ & Io8 & 73 & 86 & 223 & $47 \cdot 1$ & $I I: 26$ \\
\hline${ }_{5} \ldots \ldots \ldots \ldots 885$ & 109 & 75 & 87 & 259 & $45 \cdot 7$ & $6: 20$ \\
\hline $24 \ldots \ldots \ldots .960$ & 99 & $6 I$ & 73 & 228 & 63.0 & IO: 30 \\
\hline $25 \ldots \ldots \ldots 910$ & IOI & 60 & 71 & 232 & $67 \cdot 4$ & I $2: 24$ \\
\hline
\end{tabular}




\begin{tabular}{|c|c|c|c|c|c|c|c|}
\hline $30 \ldots \ldots \ldots$ & 1480 & 104 & 70 & 85 & 372 & $4^{8.6} 6$ & $9: 10$ \\
\hline $39 \ldots \ldots \ldots$ & 275 & I 15 & 75 & 78 & 73 & 53.0 & $3: 10$ \\
\hline $42 \ldots \ldots \ldots \ldots$ & 710 & 107 & 69 & $8 \mathrm{I}$ & 120 & $53 \cdot 7$ & $4: 45$ \\
\hline Ave.......... & 835 & I06 & 67 & 79 & $2 \mathrm{I} 4$ & $56 \cdot 7$ & $9:$ I I \\
\hline $9 \ldots \ldots \ldots$ & 195 & 129 & IOI & 108 & 52 & 27.0 & $2: 19$ \\
\hline I $8 \ldots \ldots$ & 475 & I3I & 106 & I I I & 109 & 23.1 & $2: 32$ \\
\hline Ave.......... & 335 & I30 & 103 & 109 & 80 & $25 \cdot 0$ & $2: 30$ \\
\hline $8 \ldots$ & 200 & 126 & 98 & IO4 & 53 & 29.0 & I $: 52$ \\
\hline I $7 \ldots \ldots \ldots$ & 865 & 130 & 106 & I 12 & 198 & 22.2 & $4: 5 \mathrm{I}$ \\
\hline $40 \ldots \ldots \ldots$ & 180 & 120 & 95 & IO3 & 37 & 25.8 & $3: 27$ \\
\hline Ave.......... & 415 & 125 & 100 & I06 & 96 & $25 \cdot 7$ & $3: 2$ \\
\hline
\end{tabular}

6. Yield.-The above cylinder molds were weighed empty, and immediately after filling. The cylinders were weighed just before they were tested. The amount of dry material in a given volume of paste was calculated from the figure for normal con-

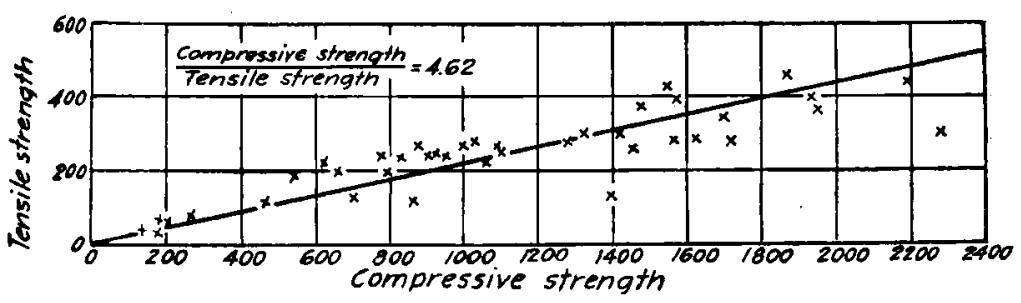

FIG. I.-Compressive strength थs. tensile strength.

sistency. These data were used to calculate the weight per cubic foot of paste, the weight of dry material per cubic foot of paste, and the weight per cubic foot of set material.

7. Tensile Strength.-Three briquettes of the usual form were made of paste of normal consistency, stored in the same way as the compressive strength specimens, and tested when one week old. The results are expressed in pounds per square inch.

The results of all of these tests are given in the accompanying tables. Owing to the extremely confusing nomenclature, no attempt has been made to group the samples according to their trade names. They have been classified, instead, in accordance with their actual compositions.

Primarily, these data are intended to enable us to arrive at numerical values expressing the different properties of gypsum. 
These values are to be used in writing specifications for the material. Incidentally, the data may also be used in an attempt to correlate the various properties. Certain efforts along this line are indicated in figures $\mathrm{I}$ and 2 , showing the relations between

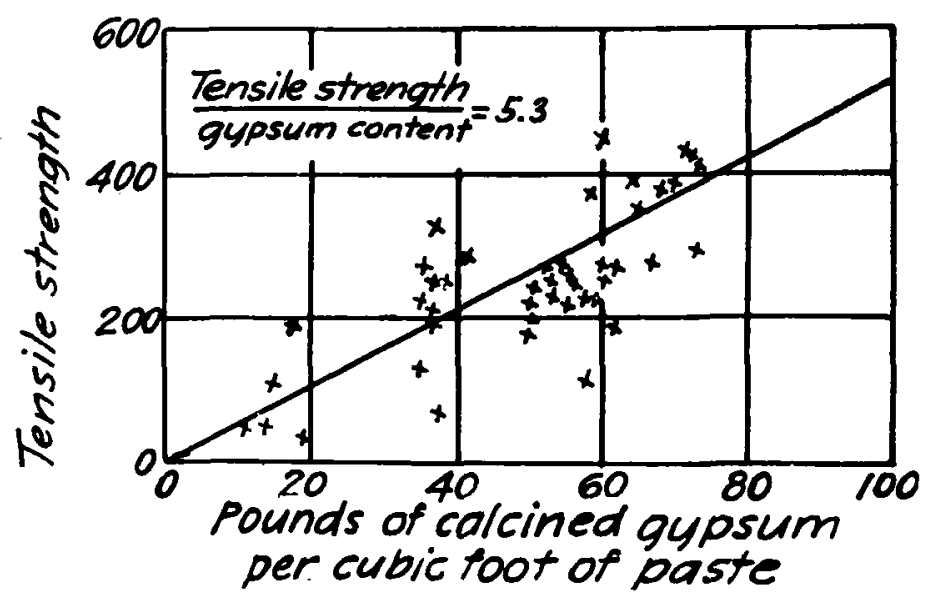

FIG. 2.-Tensile strength $v s$. gypsum content.

tensile strength and compressive strength, and tensile strength and gypsum content, respectively. Obviously the data permit and invite a great deal of this sort of calculation.

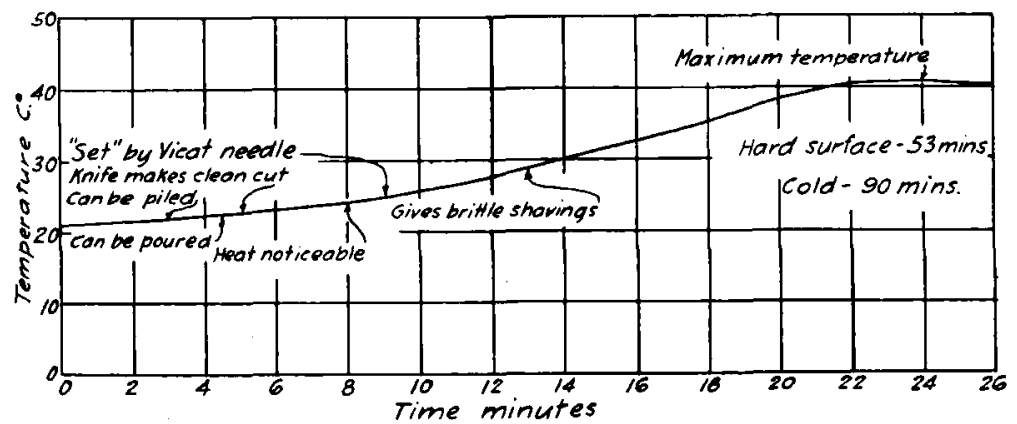

Frg. 3.-Time of set.

It seems that the present method of measuring time of set by means of the Vicat needle has been the subject of much criticism. 
To get more information on this point, the times of set of ten samples were measured by means of the temperature rise, and by direct observation. The results for one of these samples are given in figure 3. All of these results confirm those previously' obtained: that the temperature rise method is fundamentally unsound, and is misleading. The Vicat needle is endorsed. It gives results which are definite, which can be checked, and which indicate the time during which gypsum may be worked without injury.

The use of the Southard viscosimeter to measure normal consistency has also been criticized. The results obtained by means of this instrument are probably accurate within I per cent, and can be readily checked by different observers. The machine is not portable, and its use is therefore confined to the laboratory. It was suggested that a cylinder mold two inches in diameter by four inches long could easily be carried in the pocket, and a "slump" test, using this mold, would measure consistency to a sufficient degree of accuracy. Accordingly, pastes of normal consistency (by the Southard viscosimeter) were made of Io samples, and were tested by the slump method, using a two by four inch cylinder. It was found that the final diameter of the pat varied from $4^{1 / 4}$ to $5^{1 / 2}$ inches for the different samples. It would seem, therefore, that the slump method is hardly accurate enough for a standard method, although it will probably give satisfaction when used for plant control.

We wish to acknowledge our obligations to Mr. H. A. Bright for the analytical work, and to Mr. L. A. Balser for assistance in making physical measurements.

BUREaU OF STANDARDS

WaShington, D. C.

Notice-Further discussion of this subject is solicited. All communicalions should be sent to the Editor.

1 Emley, "Time of Set of Calcined Gypsum," Trans. Amer. Ceram. Soc., I9, p. 573 (1917). 